\title{
Encorafenib/Binimetinib Regimen
}

National Cancer Institute

\section{Source}

National Cancer Institute. Encorafenib/Binimetinib Regimen. NCI Thesaurus. Code C154326.

A regimen consisting of encorafenib and binimetinib that can be used for the treatment of melanoma. 